See discussions, stats, and author profiles for this publication at: https://www.researchgate.net/publication/322394665

\title{
Monocrystalline Heusler Co2FeSi alloy glass-coated microwires: Fabrication and magneto-structural characterization
}

Article in Journal of Magnetism and Magnetic Materials · January 2018

DOI: 10.1016/.jmmm.2018.01.021

CITATIONS

0

9 authors, including:

8

Ladislav Galdun

Pavol Jozef Šafárik University in Košice

5 PUBLICATIONS 6 CITATIONS

SEE PROFILE

Victor M Prida

University of Oviedo

211 PUBLICATIONS 2,279 CITATIONS

SEE PROFILE

Some of the authors of this publication are also working on these related projects:

Project Bulk Superconductors View project

Project Engineering magnetic nanowires for green technologies View project
READS

103

T. Ryba

Pavol Jozef Šafárik University in Košice

22 PUBLICATIONS 68 CITATIONS

SEE PROFILE

V. Zhukova

Universidad del País Vasco / Euskal Herriko Unibertsitatea

340 PUBLICATIONS 3,280 CITATIONS

SEE PROFILE 


\section{Accepted Manuscript}

Research articles

Monocrystalline Heusler $\mathrm{Co}_{2} \mathrm{FeSi}$ alloy glass-coated microwires: fabrication and magneto-structural characterization

L. Galdun, T. Ryba, V.M. Prida, V. Zhukova, A. Zhukov, P. Diko, V. Kavečanský, Z. Vargova, R. Varga

PII: S0304-8853(17)33744-7

DOI: https://doi.org/10.1016/j.jmmm.2018.01.021

Reference: MAGMA 63599

To appear in: Journal of Magnetism and Magnetic Materials

Received Date:

1 December 2017

Revised Date:

8 January 2018

Accepted Date:

10 January 2018

Please cite this article as: L. Galdun, T. Ryba, V.M. Prida, V. Zhukova, A. Zhukov, P. Diko, V. Kavečanský, Z. Vargova, R. Varga, Monocrystalline Heusler $\mathrm{Co}_{2} \mathrm{FeSi}$ alloy glass-coated microwires: fabrication and magnetostructural characterization, Journal of Magnetism and Magnetic Materials (2018), doi: https://doi.org/10.1016/ j.jmmm.2018.01.021

This is a PDF file of an unedited manuscript that has been accepted for publication. As a service to our customers we are providing this early version of the manuscript. The manuscript will undergo copyediting, typesetting, and review of the resulting proof before it is published in its final form. Please note that during the production process errors may be discovered which could affect the content, and all legal disclaimers that apply to the journal pertain. 


\title{
Monocrystalline Heusler $\mathrm{Co}_{2} \mathrm{FeSi}$ alloy glass-coated microwires: fabrication and magneto-structural characterization
}

\author{
L. Galdun ${ }^{1,2}$, T. Ryba ${ }^{1}$, V. M. Prida ${ }^{2}$, V. Zhukova ${ }^{3}$, A. Zhukov ${ }^{3}$, P. Diko ${ }^{4}$, V. \\ Kavečanskýn ${ }^{4}$, Z. Vargova ${ }^{1}$, R. Varga ${ }^{1, a)}$ \\ ${ }^{1}$ Inst. Phys., Fac. Sci., UPJS, Park Angelinum 9, 041 54, Kosice, Slovakia \\ ${ }^{2}$ Dpto. De Física, Universidad de Oviedo, Calvo Sotelo s/n, 33007 Oviedo, Spain \\ ${ }^{3}$ Dpto. Fisica de Materiales, Fac. Quimicas, UPV/EHU, 20009 San Sebastian, Spain \\ ${ }^{4}$ Institute of Experimental Physics, Slovak Academy of Science, Watsonova 47, 040 01, Kosice, Slovakia
}

\begin{abstract}
Large scale production of single crystalline phase of Heusler $\mathrm{Co}_{2} \mathrm{FeSi}$ alloy microwire is reported. The long microwire $(\sim 1 \mathrm{~km})$ with the metallic nucleus diameter of about $2 \mu \mathrm{m}$ is characterized by well oriented monocrystalline structure ( $B 2$ phase, with the lattice parameter $a=5.615 \AA$ ). Moreover, the crystallographic direction [101] is parallel to the wire's axis along the entire length. Additionally, the wire is characterized by exhibiting a high Curie temperature $(T c>800 \mathrm{~K})$ and well-defined magnetic anisotropy mainly governed by shape. Electrical resistivity measurement reveals the exponential suppression of the electron-magnon scattering which provides strong evidence on the half-metallic behaviour of this material in the low temperature range.
\end{abstract}

Keywords: Heusler alloy, single crystal, microwire.

\section{Introduction}

The production of spintronic devices has intensified the search for new materials, especially halfmetals, exhibiting room temperature ferromagnetism $[1,2]$. This extraordinary behaviour can be understood via distinct character of energy bands for spin up and spin down states. While spin up band overlaps the Fermi energy level $\left(E_{f}\right)$ and demonstrates a metallic character, the spin down band shows a semiconductor-like gap at the $E_{f}[3,4]$. Heusler alloys, which are well-known for their diverse and multifunctional magnetic behaviour in various fields of application, have recently attracted much attention as they are theoretically predicted to exhibit $100 \%$ spin polarization at $E_{f}[5-7]$. 
Full-Heusler alloys with the compositional stoichiometry $\left(X_{2} Y Z\right)$ crystallize in $L 2_{1}$ structure. Here $X$ and $Y$ are transition or rare earth metals (lanthanides) and $Z$ represents a main group element [8,9]. Among half-metallic alloys, Co-based full-Heusler compounds are most promising materials due to their high Curie temperatures $(T c \sim 1100 \mathrm{~K})$, high magnetic moment $\left(\sim 6 \mu_{\mathrm{B}} /\right.$ f.u. $)$ and low Gilbert damping constant $(\gamma=0.004)[10]$.

Although the $L 2_{1}$ structure is a highly ordered structure, the formation of disordered phases $(B 2, A 2$, etc.) may occur during the fabrication of alloys. The exchange between atomic positions of elements for the $X_{2} Y Z$ Heusler alloy in $L 2_{l}$ lattice can result in different structural disorders: a) exchange between $Y$ and $Z$ atoms results in $B 2$ disorder, b) exchange between $X$ and $Y$ atoms results in $\mathrm{DO}_{3}$ disorder and c) exchange between $X, Y$ and $Z$ atoms results in the $A 2$ disorder. It was predicted by theoretical calculations [11, 12], that the $Y-Z$ disorder ( $B 2$ type structure) has a much lower influence on the spin polarization in comparison to the $X-Y\left(D_{3}\right.$ type structure) and $X-Y-Z$ disorder ( $A 2$ type structure) which strongly reduces this feature.

Due to the above mentioned disorder, the production of Heusler alloys brings some disadvantages [13]. Heusler alloys are usually prepared by arc-melting method. Moreover, they require further suitable thermal treatment for a long time (from several hours up to entirely weeks) for obtaining the correct high ordered phase [14]. It was shown earlier [15], that rapid quenching by Taylor-Ulitovsky technique offers the production of glass-coated metallic microwires without the necessity of long thermal treatments. This single-step method with a high quenching rate of up to $10^{7} \mathrm{~K} / \mathrm{s}$ allows to prepare in a short time (of the order of few minutes) up to kilometres of the wire [16]. It is worth mentioning, that due to its high quenching rate it is possible to produce Heusler-based microwires with single crystalline phase and well-defined magnetic anisotropy [17-19].

In the given contribution, we present the basic structural and magnetic characterization of glasscovered Heusler $\mathrm{Co}_{2} \mathrm{FeSi}$ alloy microwires prepared by Taylor-Utilovski technique. We show that it is 
possible to produce up to kilometres of monocrystalline Heusler-based microwires in few minutes using this simple production technique that may enhance the suitability of $\mathrm{Co}_{2} \mathrm{FeSi}$ for spintronic applications.

\section{Material and methods}

The starting master alloy with nominal composition of $\mathrm{Co}_{50} \mathrm{Fe}_{25} \mathrm{Si}_{25}$ was prepared using arc-melting of pure elements $(99.99 \%)$ under argon atmosphere. Magnetic glass-coated microwires were fabricated by the Taylor-Ulitovsky technique, which consists on drawing and casting directly from the melted master alloy [20]. The diameter of inner metallic nucleus of microwire sample is around of $2 \mu \mathrm{m}$, while the diameter of external Pyrex coating is around $20 \mu \mathrm{m}$.

Chemical composition, size and shape of the microwire were characterized by scanning electron microscopy (SEM, JEOL-6100) with energy-dispersive X-ray spectroscopy (EDX) option. The microstructural and crystallographic characteristics were investigated by electron backscatter diffraction analysis (EBSD) using SEM TESCAN MIRA 3. The structure and crystalline phase were characterized by single-crystal X-ray diffraction analysis using Oxford Diffraction Xcalibur Nova diffractometer with CuK $\alpha$ radiation $(\lambda=1.5418 \AA)$. Magnetic hysteresis loops and temperature dependence of magnetization were measured on a single piece of the microwire with the length of $10 \mathrm{~mm}$ using a SQUID MPMS Quantum Design, along both, the parallel and perpendicular direction with respect to the axis of the wire, in the temperature range from $10-400 \mathrm{~K}$ and in the external fields up to $10 \mathrm{kOe}$. The resistivity was measured using standard four probe method in the temperature range from $8 \mathrm{~K}$ to $300 \mathrm{~K}$ by employing a Physical property measuring system (PPMS) Quantum Design platform. In order, to make the contacts the glass coating was released mechanically from the ends of the microwire. Afterwards, the contacts were made on metallic nucleus.

\section{Results and discussion}

Cross-section SEM image of glass-coated microwire shown in Figure 1(a) displays small metallic core with smooth surface and diameter around of $2.0 \mu \mathrm{m}$ that is covered by glass-coating with total 
diameter of $20.4 \mu \mathrm{m}$. Chemical composition of the metallic nucleus $\mathrm{Co}_{49.0} \mathrm{Fe}_{25.6} \mathrm{Si}_{25.4}$ checked by SEM EDX is in a good agreement with the expected one.
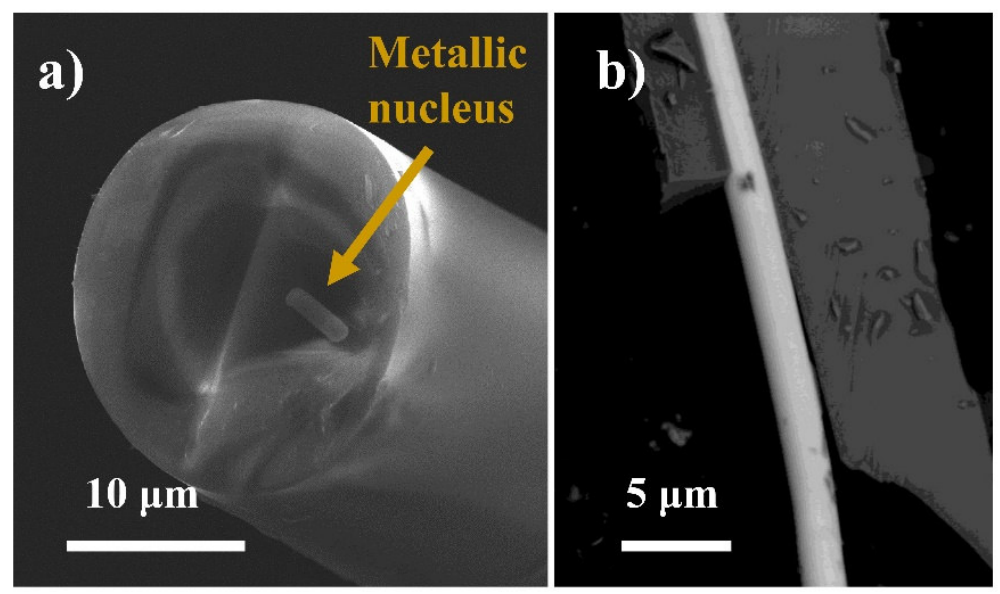

FIG.1. (a) SEM micrograph showing the cross section of a $\mathrm{Co}_{2} \mathrm{FeSi}$ glass-coated microwire. The metallic core is marked with yellow arrow and (b) SEM micrograph showing the metallic core after being released from the glass coating without any grain boundaries.

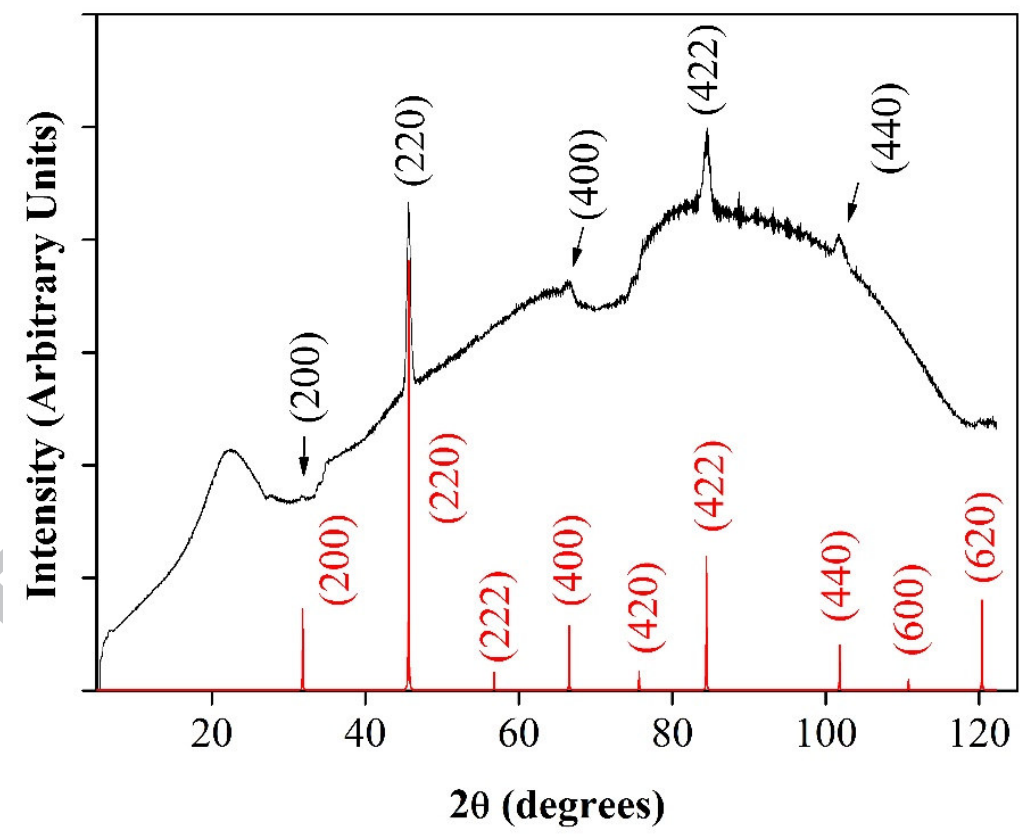

FIG.2. X-ray diffraction profile of glass-coated $\mathrm{Co}_{2} \mathrm{FeSi}$ microwire. Experimental (black) and theoretical data (red) obtained by fitting.

Figure 2 displays the $\mathrm{X}$-ray diffraction analysis of the glass-coated $\mathrm{Co}_{2} \mathrm{FeSi}$ microwires showing a wide plateau that corresponds to the glass-coating together with the crystalline patterns of the metallic 
nucleus corresponding to the $B 2$ phase with a lattice parameter $a=5.615 \AA$, which is similar to the values presented in other works (5.66 ̊) [13].

It has been shown earlier [21] that the production of glass coated microwires leads to the formation of oligocrystalline structure, where large crystals (few tens of $\mu \mathrm{m}$ ) appear along the wire. However, when the production is provided carefully with well selected diameter of the metallic nucleus and glass coating, the monocrystalline structure of the inner metallic alloy can appear. Figure 3 shows the EBSD analysis performed on an area of the surface of $\mathrm{Co}_{2} \mathrm{FeSi}$ microwire after mechanical removal of the external glass coating. Firstly, no grain boundary has been found along the entire wire. Additionally, various analysis of randomly selected pieces of the microwire revealed the same results, which points to the monocrystalline structure of entire microwire with the crystalline [101] direction lying parallel to the wire's axis. Such preferred orientation can be understood in terms of the tensile stresses induced by drawing and quenching of metallic nucleus during microwire production. During crystallization, the metallic nucleus undergoes strong axial stresses induced by the surrounding glass on the inner metallic alloy due to different thermal expansion coefficient between Pyrex and metallic nucleus.

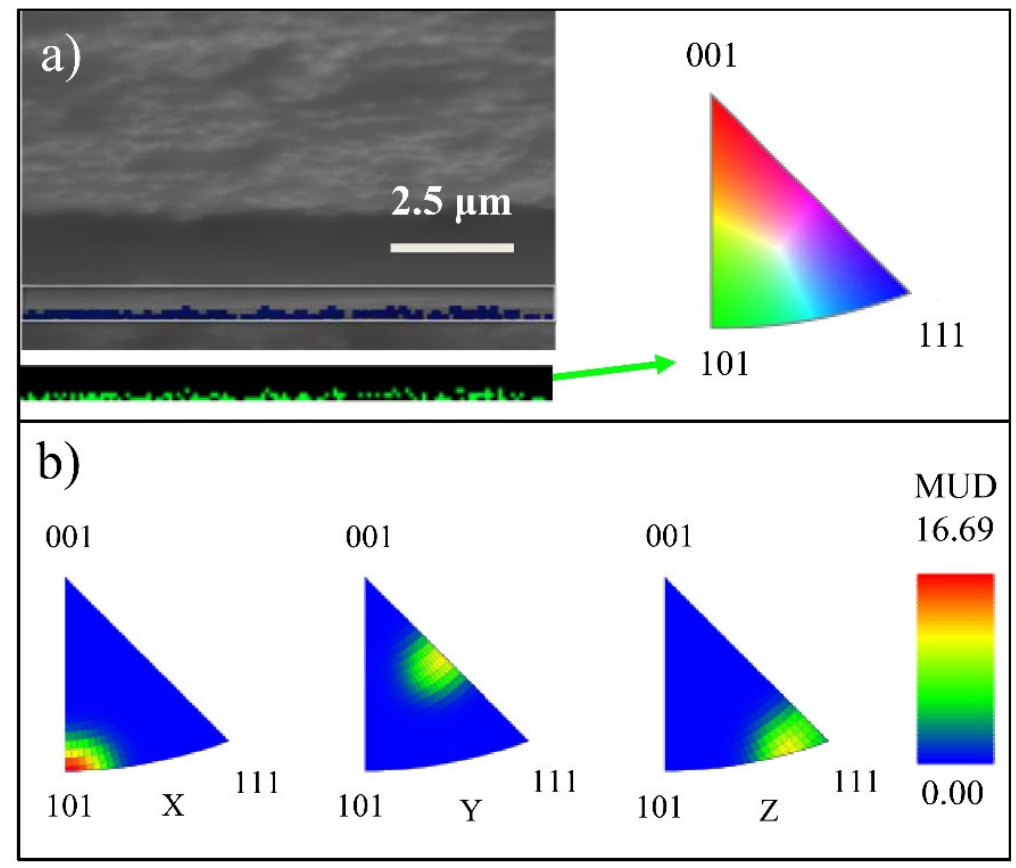


FIG.3. (a) EBSD analysis of Co2FeSi microwire and inverse pole figures (b) show monocrystalline structure with the crystallographic orientation [101] lying parallel to the microwire's axis.

Volume hysteresis loops (FIG.4) measured along both, the parallel and perpendicular direction of the applied magnetic field with respect to the wire's axis, revealed well-defined magnetic anisotropy of the metallic nucleus. The comparison between both hysteresis loops points out that the easy magnetization axis is parallel aligned to the microwire's axis. The hysteresis loop for parallel direction shows higher coercivity $(118.8 \mathrm{Oe})$ comparing to the perpendicular one $(5.6 \mathrm{Oe})$. This points to the fact that the domain wall propagation dominates in the parallel direction to the easy magnetization axis, while the hysteresis loop measured in the perpendicular direction reveals dominant magnetization rotation process (hard magnetization axis).

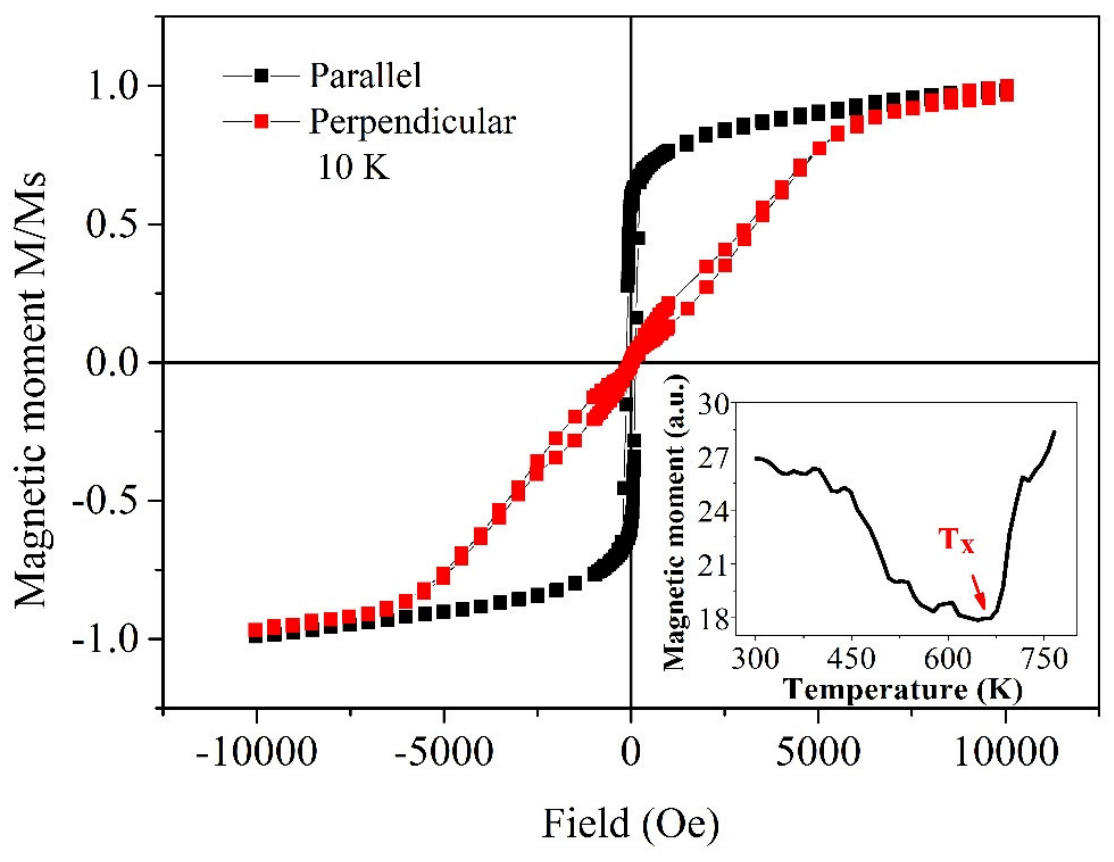

FIG. 4. Volume magnetic hysteresis loops measured at $10 \mathrm{~K}$ along the parallel (black) and perpendicular (red) directions of the applied field with respect to the wire's axis and temperature dependence of magnetization (insert) for $\mathrm{Co}_{2} \mathrm{FeSi}$ glass-coated microwire.

Temperature dependence of saturation magnetization of $\mathrm{Co}_{2} \mathrm{FeSi}$ glass-coated microwire (insert in FIG. 4) shows typical ferromagnetic behaviour with decreasing magnetization up to 640K. Above this 
temperature, magnetization starts to increase most probably due to the secondary crystallization that deteriorates the magnetic properties [15]. The irregular shape of the curve may be caused due to the annealing of the wire, which relieves the internal stresses in the metallic nucleus from the glass coating (under $640 \mathrm{~K}$ ) [22]. Moreover, the releasing of complex stresses distribution with distinct directions takes effect in the increase of the magnetization due to its rotation towards the easy magnetization axis. The Curie temperature, $T_{C}$, of $\mathrm{Co}_{2} \mathrm{FeSi}$ Heusler alloys is much higher than crystallization temperature $\left(T_{x}=670 \mathrm{~K}\right)$ and higher than our measuring interval $\left(T_{C} \sim 1100 \mathrm{~K}[10]\right)$.

As previously mentioned in the introduction, the $B 2$ phase is a $Y-Z$ disordered structure, which may reduce the values of spin polarization. However, recent theoretical investigation on the spin polarization of fully ordered $L 2_{1}$ and fully disordered $B 2$ structure of $\mathrm{Co}_{2} \mathrm{FeSi}$ Heusler alloy reveals that both systems are half metallic. [2]

Lately studies [13, 23-26] have shown that the half-metallic character and structural disorder of Heusler alloys can be predicted by simple electrical resistivity variation with temperature. In our case, the resistivity data was found to fit well with the relations presented in the published work by Bombor et $a l$, [26]. By taking into account the predicted half-metallic character of the $B 2$ disordered phase of $\mathrm{Co}_{2} \mathrm{FeSi}$ Heusler alloy, the absence of minority spin states at Fermi level, $E_{F}$, implies the existence of an energy gap $\Delta$ for only one spin direction.

In ordinary ferromagnets the resistivity, $\rho$, shows a characteristic $\sim \mathrm{T}^{2}$ dependence that arises from electron-magnon (i.e., spin-flip) scattering. In the case of half-metallic ferromagnets, the spin flips of conduction electrons should be suppressed due to the energy gap $\Delta$. Therefore, the characteristic $\sim \mathrm{T}^{2}$ dependence is expected to be exponentially suppressed within the temperature range where $\mathrm{k}_{\mathrm{B}} \mathrm{T} \leq \Delta$. Additionally, the resistivity of a metal is usually dominated by electron-phonon scattering characterized by a linear temperature dependence at elevated temperatures $(\mathrm{T} \geq 50 \mathrm{~K})$. 
The attempt to fit resistivity variation with temperature, $\rho$ (T) (FIG. 5), of the monocrystalline $\mathrm{Co}_{2} \mathrm{FeSi}$ metallic nucleus with quadratic and quadratic plus linear temperature dependence fails completely. Therefore, we fitted resistivity with combined scattering of residual $\left(\rho_{R}\right)$, magnonic $\left(\rho_{M}\right)$, and phononic $\left(\rho_{\mathrm{P}}\right)$ contributions according to previous study [26]:

$$
\rho=\rho_{R}+\rho_{M}(T)+\rho_{P}(T) .
$$

The residual resistivity $\left(\rho_{R}\right)$ is temperature independent and is caused by the defects of the crystal lattice or additional strong stresses induced by the glass-cover on metallic nucleus. The usually quadratic magnonic term $\rho_{M}(T)$ is expected to be exponentially suppressed because of the gapped spin-flip scattering [26]. The resistivity data in the low temperature interval ( $\mathrm{T} \leq 88 \mathrm{~K}$ ) was found to fit well with the electron-magnon scattering dependence empirically weighted by a Boltzmann factor [26],

$$
\rho_{M}(T)=C T^{2} \exp \left(\frac{-\Delta}{k_{\mathbf{B}} T}\right) \text {. }
$$

The parameter $\mathrm{C}$ presents the measure of the strength of the magnon scattering process. The phononic part of the scattering process $\left(\rho_{\mathrm{P}}\right)$ is well described by the Bloch-Grüneisen formula,

$$
\rho_{P}(T)=A\left(\frac{T}{\Theta_{D}}\right)^{5} \int_{0}^{\Theta_{D} / T} \frac{x^{5}}{\left(e^{x}-1\right)\left(1-e^{-x}\right)} d x
$$

The resistivity fit yields the Debye temperature $\Theta_{D}=330 \mathrm{~K}$ and an energy gap

$$
\Delta / k_{B}=37 \mathrm{~K},(\Delta=3.2 \mathrm{meV}) .
$$

The estimated value of the Debye temperature is in a good agreement with the one obtained in a previous study [26]. On the other hand, the extracted gap size is smaller as compared to the theoretical calculations by more than one order and is also in a good agreement with previous experimental results [26]. In spite of that, the observed experimental suppression of spin-flip scattering confirms the halfmetallic character. However, the extracted value of $\Delta$ points to the fact, that the $100 \%$ spin polarization of the conduction electron on the Fermi level is present only for the temperature interval $\mathrm{k}_{\mathrm{B}} \mathrm{T}<\Delta$. 


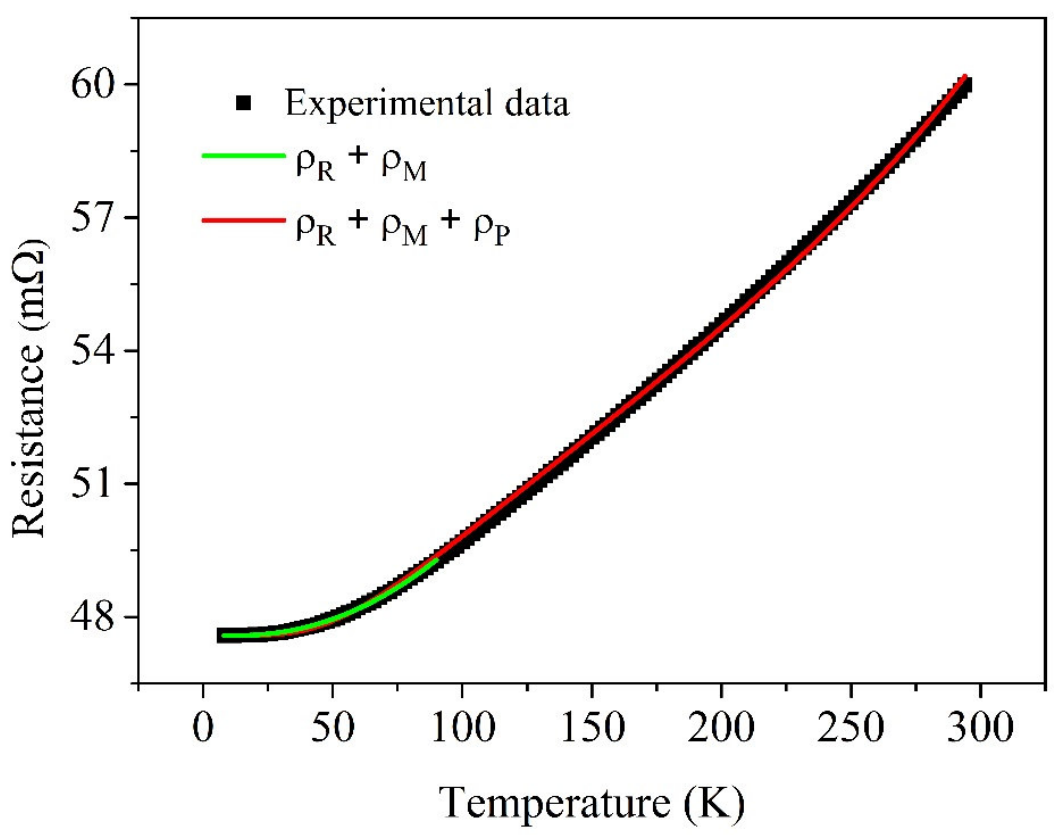

FIG. 5. Temperature dependent electrical resistivity for $\mathrm{Co}_{2} \mathrm{FeSi}$ glass-coated microwire. The experimental values are displayed as black line. While the green line represents the fit of $\sim \mathrm{T}^{2}$ magnonic dependence with the additional Boltzmann factor, the red line shows the fit with magnonic-phononic temperature dependence.

\section{Conclusion}

In conclusion, we report on fabrication and characterization of monocrystalline Heusler $\mathrm{Co}_{2} \mathrm{FeSi}$ glass-coated microwire prepared by Taylor-Ulitovsky technique. The wire is characterized by monocrystalline $B 2$ structure $(a=5.615 \AA)$ along the entire length, which is characterized by the mutual disorder between $Y(\mathrm{Fe})$ and $Z(\mathrm{Si})$ atoms. Additionally, above mentioned monocrystalline structure is well oriented having [101] crystallographic direction parallel to the wire's axis. Apart from high Curie temperature, such a wire shows well-defined magnetic anisotropy with the easy magnetization axis parallel to the wire's axis. The electrical transport measurement points to the half-metallic features of this ferromagnetic material. However, half-metallic behaviour was only observed in the temperature range of $\mathrm{T} \leq 88 \mathrm{~K}$. Taking into account that Taylor-Ulitovsky method allows production of wire with the diameter down to $90 \mathrm{~nm}$ [27], it is clear that easy and low-cost preparation of large amount of 
monocrystalline Heusler-type microwires offers new possibilities in spintronic applications, where materials with high spin polarization is desired.

\section{Acknowledgements}

The authors thank to Prof. A. Feher for the fruitful discussion. This work was supported by NanoCEXmat ITMS 26220120035, Slovak Grant Agency [grant number VEGA 1/0060/13], Slovak Grant Agency [grant number APVV-16-0079], and Slovak Grant Agency [grant number APVV-002711]. Spanish MINECO research projects [grant number MAT2013-48054-C2-2-R] and [grant number MAT2016-76824-C3-3-R], together with FICyT and Consejería de Economía y Empleo from Principality of Asturias under project [grant number GIC-FC-15-GRUPIN14-085] are gratefully acknowledged. The technical assistance provided by the Scientific-Technical Services (SCTs) of the University of Oviedo is also recognized.

\section{References}

1. X. Zhu, Y. Wang, L. Wang, Y. Dai, Ch. Luo, Pressure and disorder effect on the half-metallic character and magnetic properties of the full-Heusler alloy $\mathrm{Co}_{2} \mathrm{FeSi}$, J. Phys. Chem. Solids 75 (2014) 391-396.

2. R. Mohankumar, S. Ramasubramanian, M. Rajagopalan, M.M. Raja, S. V. Kamat, J. Kumar, Density functional study of half-metallic property on $\mathrm{B} 2$ disordered $\mathrm{Co}_{2} \mathrm{FeSi}$, J. Mater. Sci. 50 (2015) $1287-1294$.

3. J. Li, F. Meng, G. Liu, X. Chen, L. Hongzhi, E. Liu, G. Wu, Electronic structure and magnetism of binary Fe-based half-Heusler alloys $\mathrm{Fe}_{2} \mathrm{Z}(\mathrm{Z}=\mathrm{In}, \mathrm{Sn}, \mathrm{Sb}$, and As), J. Magn. Magn. Mater. $331(2013) 82-87$. 
4. N. Nazmunnahar, T. Ryba, J.J. del Val, M. Ipatov, J. Gonzalez, V. Hašková, P. Szabó, P. Samuely, J. Kravcak, Z. Vargova, R. Varga, Half-metallic $\mathrm{Ni}_{2} \mathrm{MnSn}$ Heusler alloy prepared by rapid quenchung, J. Magn. Magn. Mater. 386 (2015) 98-101.

5. M. Singh, H.S. Saini, J. Thakur, A.H. Reshak, M.K. Kashyap, Tuning Fermi level of $\mathrm{Cr}_{2} \mathrm{CoZ}$ (Z = $\mathrm{Al}$ and $\mathrm{Si}$ ) inverse Heusler alloys via Fe-doping for maximum spin polarization, J. Magn. Magn. Mater. 370 (2014) 81-86.

6. L. Makinistian, M.M. Fiaz, R.P. Panguluri, B. Balke, S. Wurmehl, C. Felser, E.A. Albanesi, A. G. Petukhov, B. Nadgorny, On the half-metallicity of $\mathrm{Co}_{2} \mathrm{FeSi}$ Heusler alloys: Point-contact Andreev reflection spectroscopy and ab initio study, Phys. Rev. B 87 (2013) 220402.

7. J.-P. Wüstenberg, M. Cinchetti, M. S. Albaneda, M. Bauer, M. Aeschlimann, Spin- and timeresolved photoemission studies of thin $\mathrm{Co}_{2} \mathrm{FeSi}$ Heusler alloy films, J. Magn. Magn. Mater. 316 (2007) 411-414.

8. T. Graf, C. Felser, S.S.P. Parkin, Simple rules for the understanding of Heusler compounds, Prog. Solid State Ch. 39 (2011) 1-50.

9. T. Ryba, Z. Vargova, J. Kovac, P. Diko, V. Kavečanský, S. Piovarči, C. Garcia, R. Varga, Structural and magnetic characterization of half-metallic $\mathrm{Co}_{2} \mathrm{MnAl}$ Heusler alloy, IEEE Trans. Magn. 51 (2015) 2600103. 
10. S. Wurmehl, G. H. Fecher, H. Ch. Kandpal, V. Ksenofontov, C. Felser, Geometric, electronic, and magnetic structure of $\mathrm{Co}_{2} \mathrm{FeSi}$ : Curie temperature and magnetic moment measurements and calculations, Phys. Rev. B 72 (2005) 184434.

11. Y. Miura, K. Nagao, M. Shirai, Atomic disorder effects on half-metallicity of the full-Heusler alloys $\mathrm{Co}_{2}\left(\mathrm{Cr}_{1-\mathrm{x}} \mathrm{Fe}_{\mathrm{x}}\right) \mathrm{Al}$ : A first-principles study, Phys. Rev. B 69 (2004) 144413.

12. Z. Gercsi, K. Hono, Ab initio predictions for the effect of disorder and quarternary alloying on the half-metallic properties of selected $\mathrm{Co}_{2} \mathrm{Fe}$-based Heusler alloys, J. Phys.: Cond. Matt. 19 (2007) 326216.

13. K. Srinivas, M. M. Raja, S. V. Kamat, Effect of partial substitution of silicon by other sp-valent elements on structure, magnetic and electrical resistivity of $\mathrm{Co}_{2} \mathrm{FeSi}$ Heusler alloys, J. Alloys Comp. 619 (2015) 177-185.

14. P. J. Brown, A. P. Gandy, R. Kainuma, T. Kanomata, T. Miyamoto, M. Nagasako, K. U. Neumann, A. Sheikh, K. R. A. Ziebeck, Atomic order and magnetization distribution in the half metallic and nearly half metallic C1b compounds NiMnSb and PdMnSb, J. Phys.-Condens. Mat. 22 (2010) 206004.

15. L. Galdun, T. Ryba, V.M. Prida, B. Hernando, V. Zhukova, A. Zhukov, Z. Vargova, R. Varga, $\mathrm{Ni}_{2} \mathrm{FeSi}$ Heusler glass coated microwires, Acta Phys. Pol. A 131 (2017) 851-853. 
16. R. Varga, T. Ryba, K. Saksl, V. Zhukova, J. Gonzalez, A. Zhukov, Studies of magnetic and structural properties of Ni-Mn-Ga Heusler-type microwires, J. Optoelectron. Adv. M. 14 (2012) 257-261.

17. R. Varga, T. Ryba, Z. Vargova, K. Saksl, V. Zhukova, A. Zhukov, Magnetic and structural properties of Ni-Mn-Ga Heusler type microwires, Scripta Mater. 65 (2011) 703-706.

18. M.I. Ilyn, V. Zhukova, J. D. Santos, M.L. Sánchez, V.M. Prida, B. Hernando, V. Larin, J. González, A.M Tishin, A. Zhukov, Magnetocaloric effect in nanogranular glass coated microwires Phys. Stat. Sol. (a) 205 (2008) 1378-1381.

19. V. Vega, L. González, J. García, W.O. Rosa, D. Serantes, V.M. Prida, G. Badini, R. Varga, J.J. Sunol, B. Hernando, Ni59.0Mn23.5In17.5 Heusler alloy as the core of glass-coated microwires: Magnetic properties and magnetocaloric effect J. Appl. Phys. 112 (2012) 033905-1-8.

20. H. Chiriac, T.-A. Óvári, Amorphous glass-covered magnetic wires: Preparation, properties applications, Progress Mater. Science 40 (1996) 333-407.

21. P. Zheng, N.J. Kucza, Ch.L. Patrick, P. Müllner, D.C. Dunand, Mechanical and magnetic behavior of oligocrystalline Ni-Mn-Ga microwires, J. Alloys Comp 624 (2015) 226-233.

22. H. Chiriac, T.A. Óvári, Gh. Pop, Internal stress distribution in glass-covered amorphous magnetic wires, Phys. Rev. B 52 (1995) 10104-10113. 
23. M. A. Kouacou, A.A. Koua, Z. Yeo, A. Akichi, A. Tanoh, M. Koffi, Magnetic properties and electrical resistivity of metallic ferromagnetic compounds as the Half Heusler PtMnSb, J. Applied Sci. 8 (4) (2008) 682-686.

24. C.G.F. Blum, C.A. Jenkins, J. Barth, C. Felser, S. Wurmehl, G. Friemel, C. Hess, G. Behr, B. Büchner, A. Reller, S. Riegg, S.G. Ebbinghaus, T. Ellis, P.J. Jacobs, J.T. Kohlhepp, H.J.M. Swagten, Highly ordered, half-metallic Co2FeSi single crystals, Appl. Phys. Lett. 95 (2009) 161903.

25. G. Prathiba, S. Venkatesh, M. Rajagopalan, N. H. Kumar, Half metallic $\mathrm{Co}_{2} \mathrm{TiGe}-\mathrm{a}$ theoretical and experimental investigation, J. Magn. Magn. Mater. 323 (2011) 22-27.

26. D. Bombor, Ch. G. F. Blum, O. Volkonskiy, S. Rodan, S. Wurmehl, Ch. Hess, B. Büchner, Half-Metallic ferromagnetism with unexpected small spin splitting in Heusler Compound $\mathrm{Co}_{2} \mathrm{FeSi}$, Phys. Rev. Lett. 110 (2013) 066601.

27. T.-A. Óvári, H Chiriac, Domain wall mobility in rapidly solidified ultrathin amorphous wires, J. Appl. Phys. 113 (2013) 17A304. 